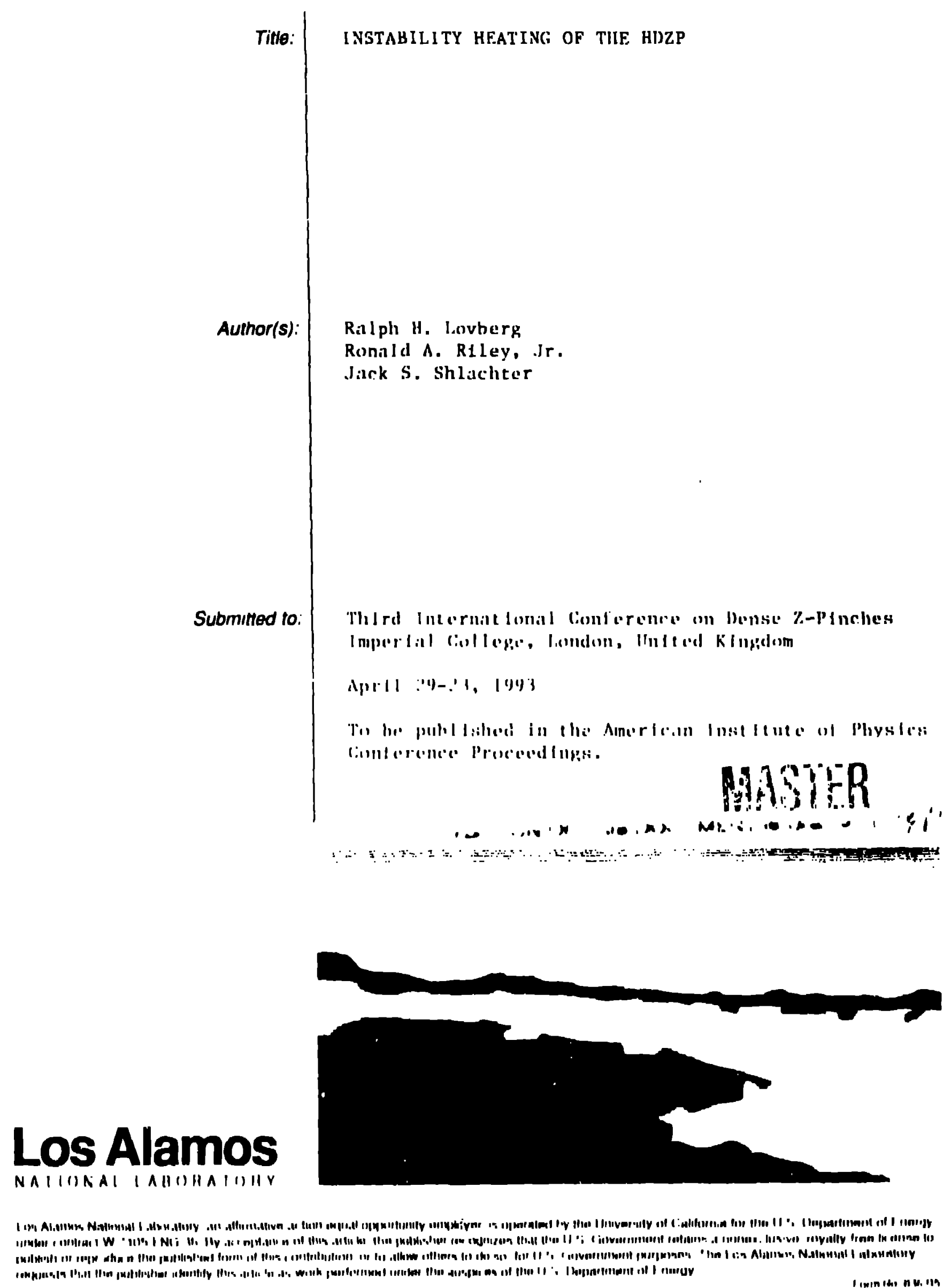




\title{
INSTABILITY HEATING OF THE HDZP
}

\author{
R.H. Lovberg \\ University of California San Diego, La Jolla, CA 92093 \\ R.A. Riley, and J.S. Shlachter \\ I os Alamos National Laboratory, Los Alamos, NM 87545
}

\begin{abstract}
We present a model of dense Z-Pinch heating. For pinches of sufficiently small diameter and high current, direct ion heating by $\mathbf{m}=0$ instabilities becomes the principal channel for power input. This process is particularly important in the present generation of dense micro-pinches (e.g., HDZP-II) where instability growth times are much smaller then current risetimes, and a typical pinch diameter is several orders smaller than that of the chamber. Unde- the se cu aditions, $m=0$ formation is not disruptive: the large $E_{z}$ field reconnects the instability cusps externally, after which the ingested magnetic flux decays into turbulent kinetic energy of the plasma. The continuous process is analogous to boiling of a heated fluid.

$A$ simple analysis shows that an equivalent resistance

$$
R_{r}-\frac{l}{4 \sqrt{N m_{i}}}\left(\frac{\mu_{0}}{\pi}\right)^{3 / 2} \frac{l}{r}
$$

appears in the driving circuit, where I is the pinch current. $\mathbf{N}$ is the line density, $\ell$ is the pinch leength, $m_{i}$ is the ion mass, and $r$ is the pinch radius. $A$ corresponding heating term has been added to the ion energy equation in a 0-I). self-similar simulation, which had heen written previously to estimate fusion yields and tadial expansion of $\mathbf{D}_{2}$ fiber pinches. The simulation results agree well with the experimental results from HDYP-II. where the assumption

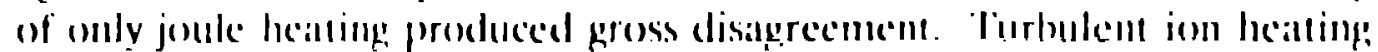

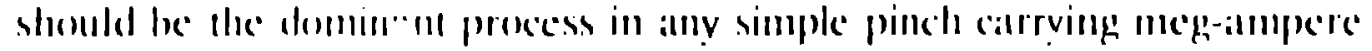
current and hat ing? submillimeter radlus.
\end{abstract}

\section{INTKOI)IC:TTON}

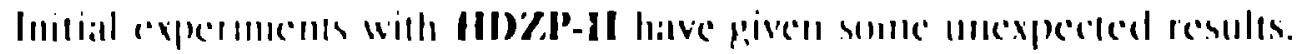
Amomy! the'm ins:

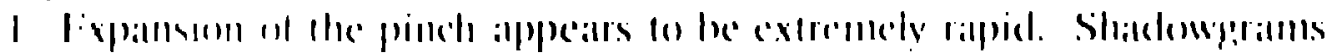

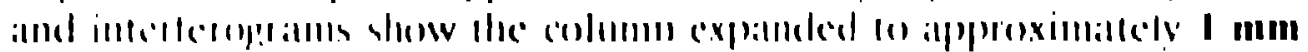

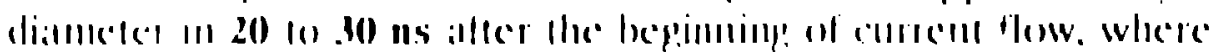

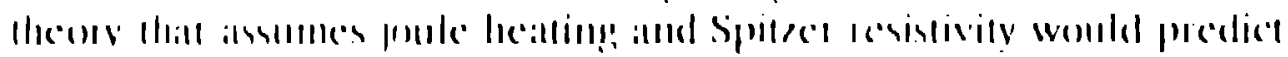


only slight expansion. Fig. 1 is an interferogram of the pinch taken 20 ns after the onset of current. At this time, the pinch diameter appears to be slightly greater than $1 \mathrm{~mm}$.

2. Neutron yield is two orders of magnitude smaller than would have been expected for the parameters of the experiment.

3. Intense $m=0$ instability appears from the earliest times at which images have been obtained; it does not appear to disrupt the pinch. However, $\mathbf{m}=\mathbf{1}$ instability has not been observed.

In this paper, we make an estimate of the effect of direct plasma heating by $\mathbf{m}=\mathbf{0}$ instability growth and show that the observed expansion and radiation benavior can plausibly arise from it.

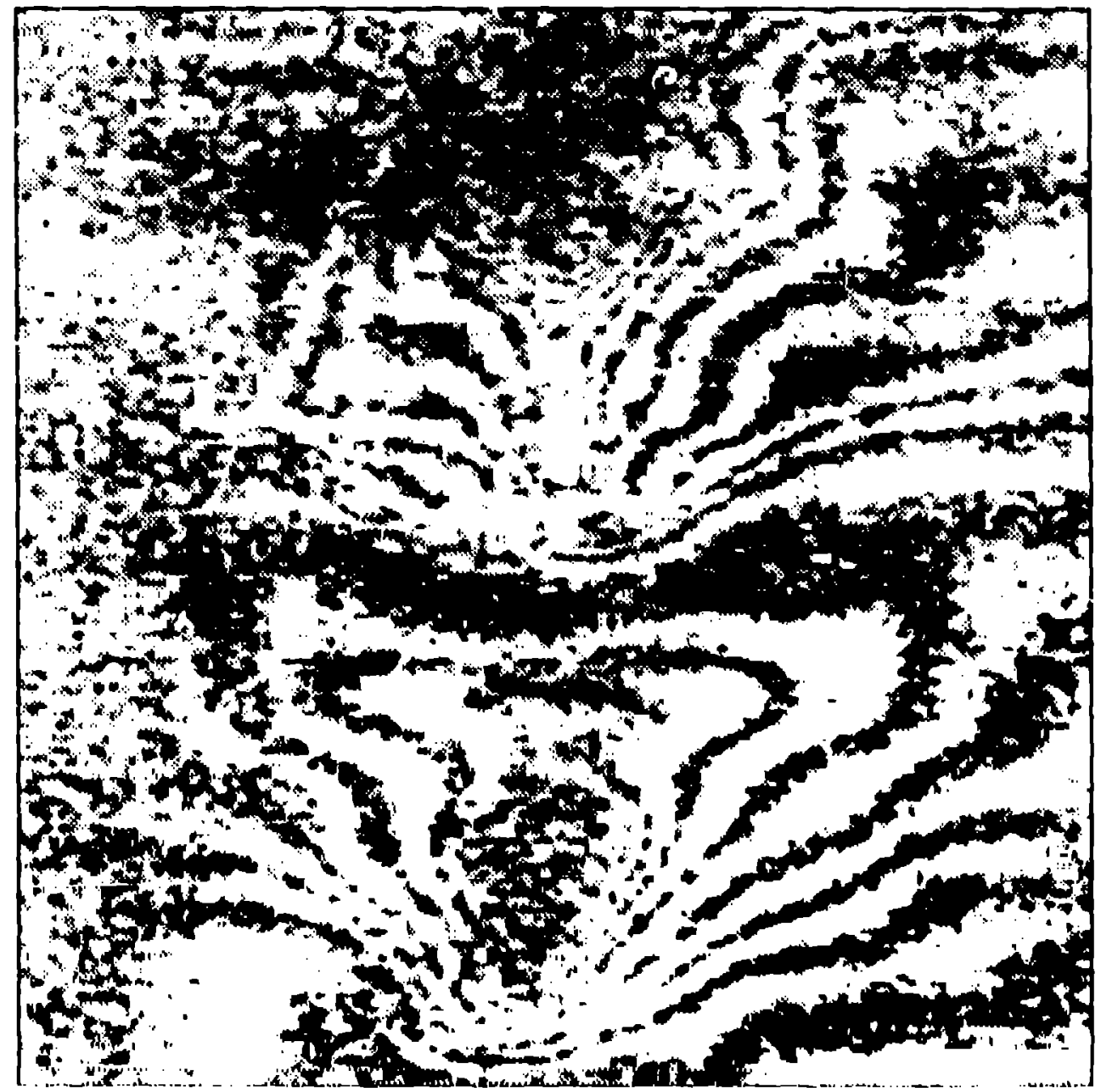

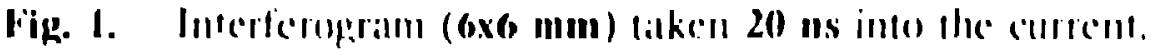

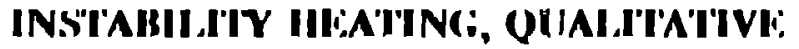

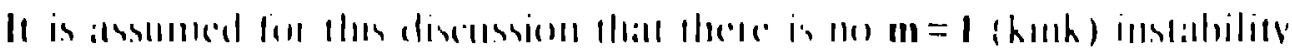

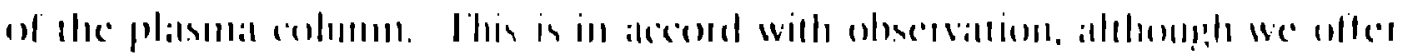

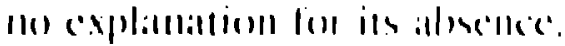


When $\mathbf{m}=\mathbf{0}$ deformations occur, the plasma acquires a macroscopic fluid-kinetic energy associated with deformation of its original cylindrical shape. In the nonlinear limit of the growth of the $\mathbf{m}=\mathbf{0}$ mode, circular cusps of plasma are ejected radially away from the pinch (Fig. 2). In early experiments with gas pinches, these cusps generally reached the discharge tube wall, so that full development of instability growth usually resulted in disruption and termination of the discharge. In the HDZP-II, radially ejected plasma is still very far from the wall, so no disruption occurs. Moreover, the applied electric field in the HDZP-II remains strong for hundreds of instability growth times, so that one should expect an external reconnection of the cusps,

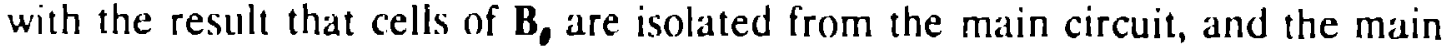
current is diverted to the region outside of the cusps. The total energy contained within the larger pinch now consists of ion and electron thermal energy, turbulent kinetic energy of ions, and isolated cells of magnetic energy.

A detached torus of tlux within the plasrna has no equilibrium state short of a collapse of its inner interface with the plasma to zero radius. In reaching this limit, it will have given up most of its magnetic energy to work against the plasma, and as a result the ions receive most of this energy.

This process then continually iepeats itself, amounting to a virtual "boiling" of the pinch.

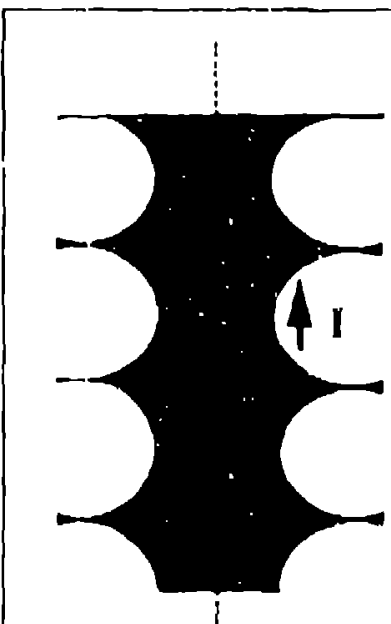

(d)

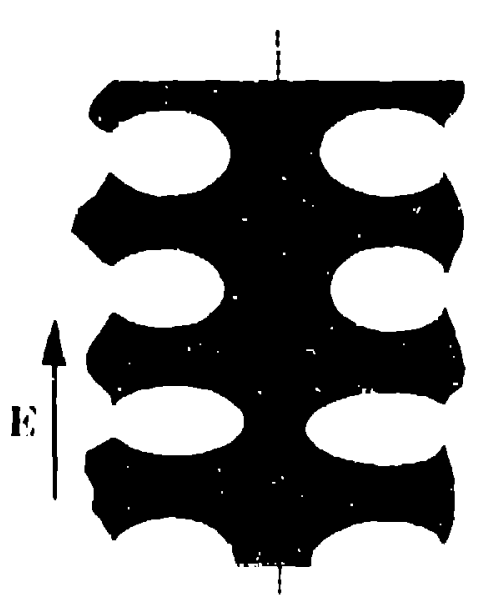

(b)

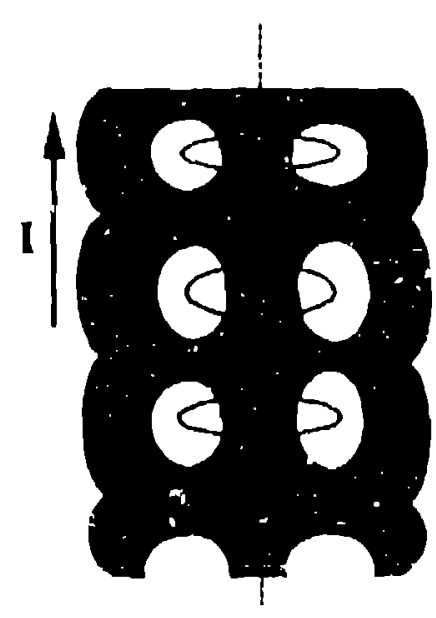

(c)

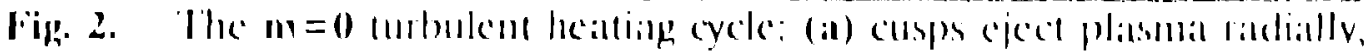

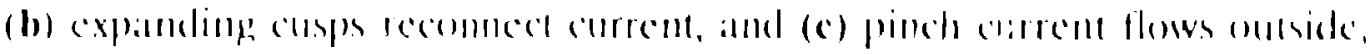
1! alperel collis ot 18

\section{INSE'IAISIIIIY IIEA'TING, OHANIITA'TIVE:}

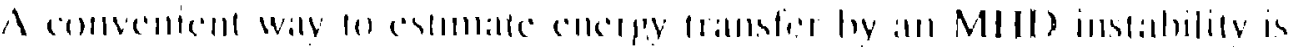

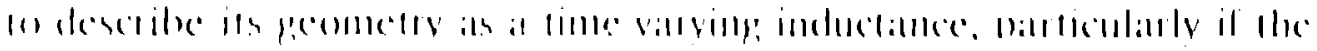

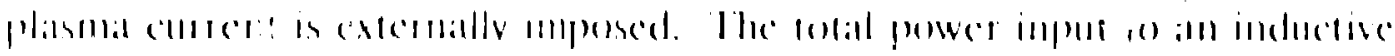


circuit for which resistance is negligible is

$$
P=I V=I \frac{d}{d t}(L I)=I L \frac{d I}{d t}+I^{2} \frac{d}{d t} L
$$

A slight rearrangement separates the terms functionally:

$$
P=\frac{d}{d t}\left(\frac{1}{2} L J^{2}\right)+\frac{I^{2}}{2} \frac{d L}{d t}
$$

The second term represents the power input to gross kinetic energy of the deformable conductor, while the first term is the rate of increase of magnetic energy.

The $m=0$ instability raises the inductance of sections of the pinch through a shrinking of the inner coaxial conductor (plas!mai) diameter. The inductance of such a pinch section with radius $r$, length $\ell$, and wall radius $b$ is

$$
L: \frac{\mu_{0} l}{2 \pi} \ln \left(\frac{h}{r}\right) \text {, }
$$

so that the rate of inductance change associated with pinch radius change is

$$
\frac{d L}{d t}--\frac{\mu_{0} \ell}{2 \pi r} \frac{d r}{d t} \text {. }
$$

I:equating the radial velocity of the pinch on the lecal Alfven speed.

$$
\frac{d r}{d t}=\nu_{d}=\frac{B_{0}}{\sqrt{\mu_{0} \rho}},
$$

expressing 13 , in terms of current and radius,

$$
B_{0} \quad \begin{aligned}
& \mu_{0} I \\
& 2 \pi r
\end{aligned}
$$

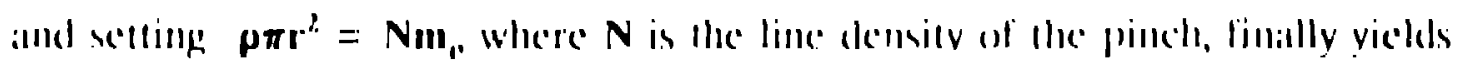

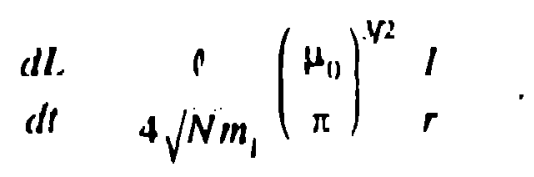

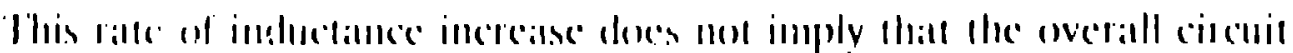

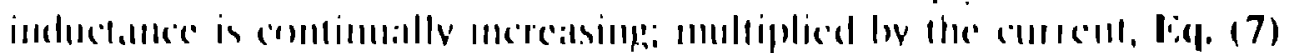

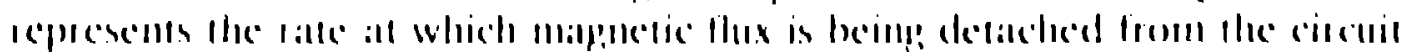

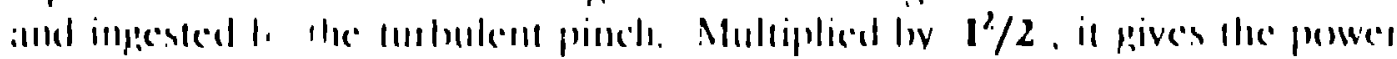

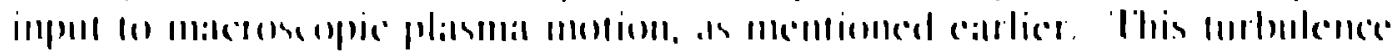

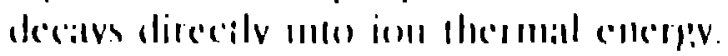


The inductance decrease associated with the outward moving cusps is small compared to the increase from the necks, if the motion is assumed to be approximately volume-conserving. The rate of inductance decrease associated with the overall expansion of the pinch is negligible, since the expansion goes at about $2 \%$ of the Alfvéll speed.

The rate of loss of magnetic energy from the circuit through ingestion into the pinch is contained in the first right-hand term of Eq.(2). Since, in the unstable motion, the relative growth rate of inductance is far greater than that of the current, it is reaconable to approximate I as constant over an instability growth time. In this case, the two right terms in Eq.(2) become identical, so the total power into ion: is

$$
P_{1}=I^{2} \frac{d L}{d t}
$$

\section{PINCH SIMULATION}

This heating term has been incorporated into a 0-D pinch simulation. winch was modified from one written previously to study the effects of alphaparticle retention at larjec burn rates. This code, BURN4, assumes selfsimularity of the plasma profile during radius change. It incorporates joule heating, bremsstrahlung radiation, energy exchanges among various particle yecies, and gas dynamics tha!, as is usual under the self-similar assumption, alssume the instantaneous transmission of pressure changes through the pinch.

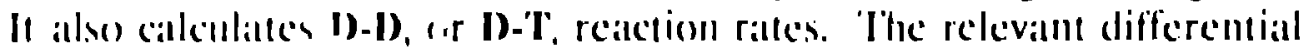
cyuations are given in Appendix A.

The new leating term is added to the ion energy ecpuation. An arbitrary "ness must be mide of the fraction of the whole pinch length undergoing instable radial motion at any instant. Based on analysis of a mumber of images showing $\mathbf{m}=0$ activity, we provisionally set this fraction lo 0.1.

\section{SIMUI A'TION RHSUI:IX}

For complarison, tums were made with and withent the instability healling

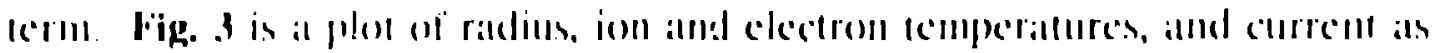
a function of timle, with ille simusoidal corrent waveform adjusted to the same

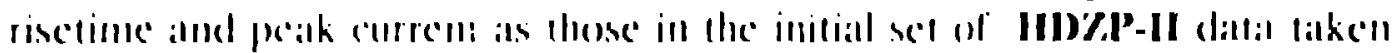

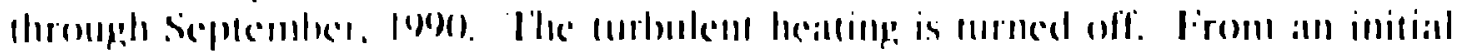

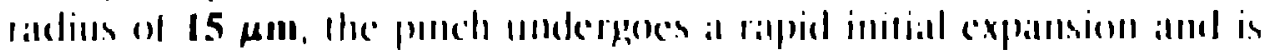

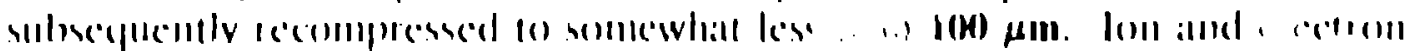

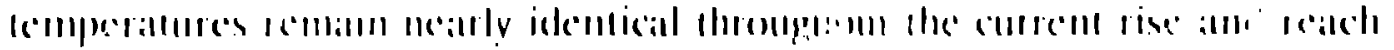

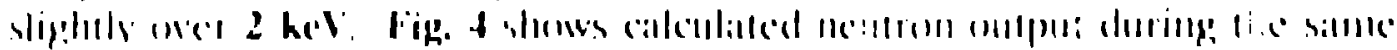

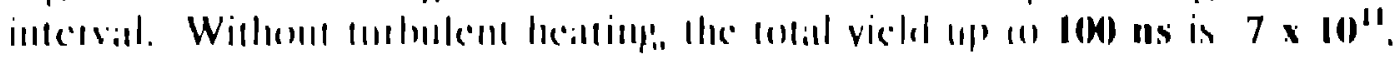

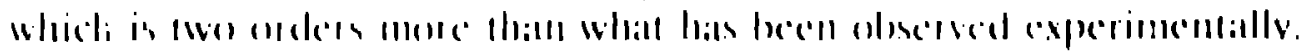




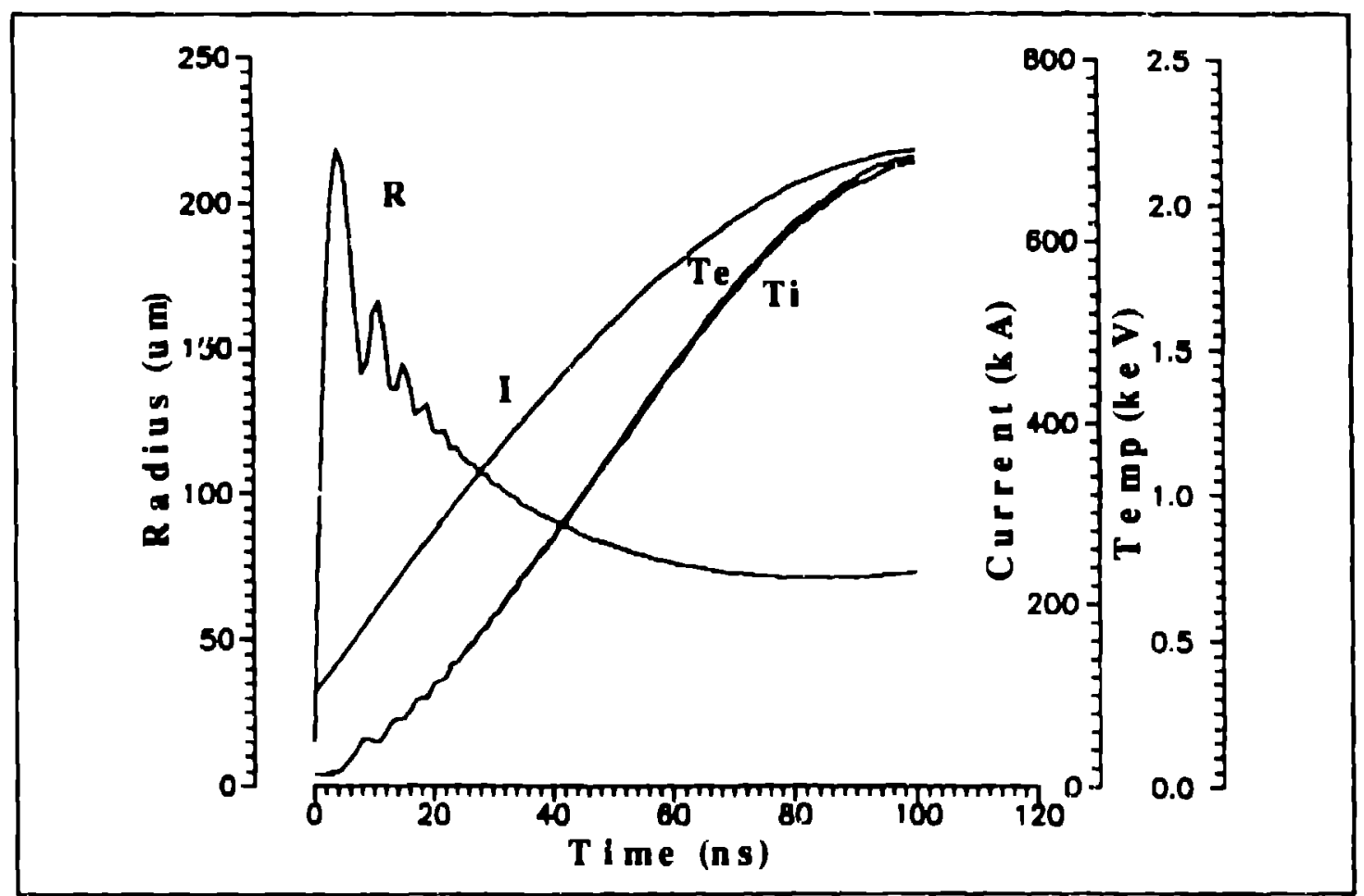

Fig. 3. Joule-heated HDZP-II 0-D simulation results.
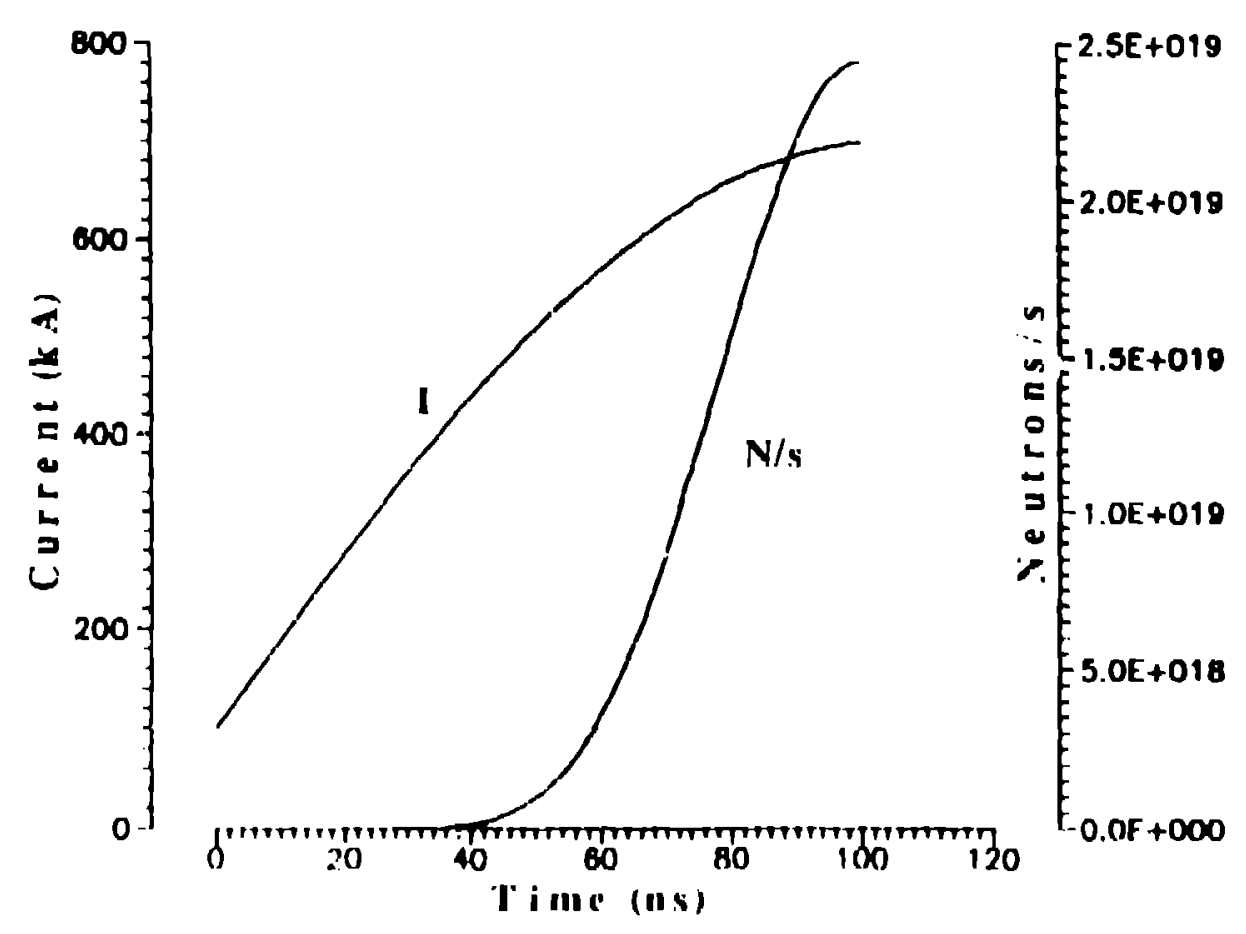

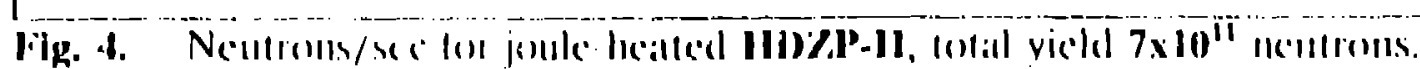


In contrast, results of the simulation with turbulent heating turned on are remarkably consistent with observation. Fig. 5 shows the radius increasing to nearly $3 \mathrm{~mm}$ during the current rise. At $30 \mathrm{~ns}$, it has reached a radius of $500 \mu \mathrm{m}$, which is its approximate value in the interferogram of Fig. 1 . Ion temperature is nearly $4 \mathrm{keV}$ at $100 \mathrm{~ns}$, while the electrons remain fairly cold, not exceeding $700 \mathrm{eV}$. The very rapid plasma expansion decouples ions and electrons, and the joule heating of electrons can barely supply the temperature lost to adiabatic decompression.

The neutron yield for this case is $8 \times 10^{9}$, which is in the range of yields obtained experimentally.

The low electron temperature can explain some difficulty in obtaining unambiguous soft $x$-ray signals at low-keV energies.

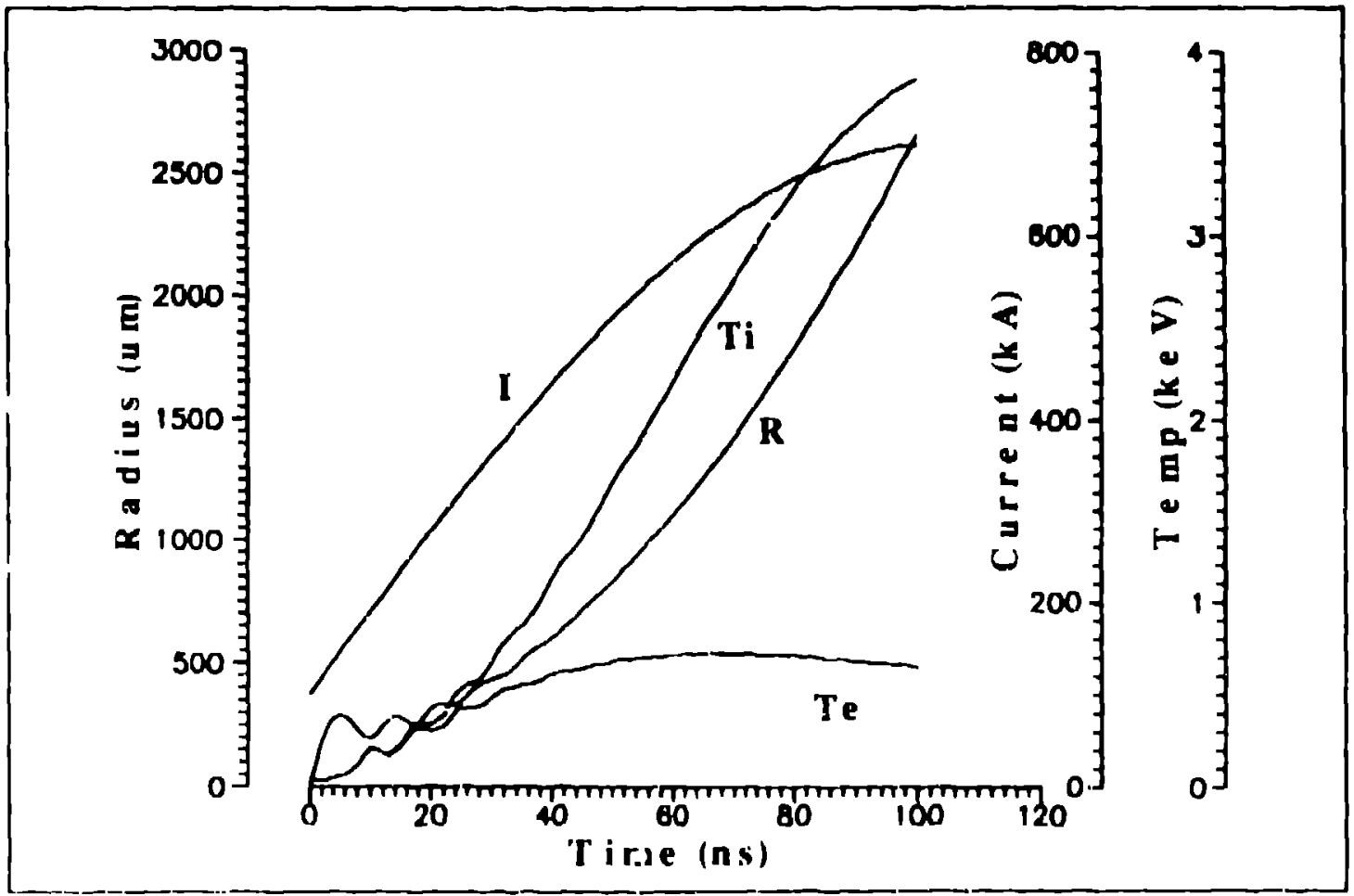

lïg. 5 . The $m=0$ turbulent-heated HDzP-II 0-D simulation results.

\section{IMPURITY ADMIXTURE}

(1)e experiment in the series that generated the datal pusted above involved loading the D, liber with approximately low; of acon. The mentive wis (1) produce sipnificant carly-time radiation loss that would inhibit the

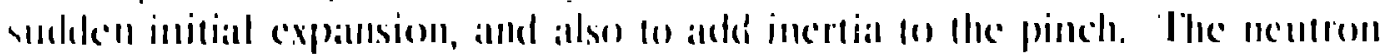

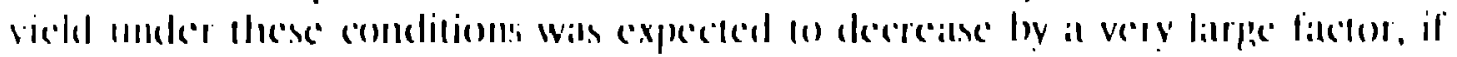
III) (1) disalppear allogethes.

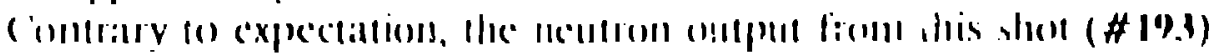


remained at the level of the pure $\mathbf{D}_{2}$ shots. This result cannot be understood if one supposes that ion heating is through transfer frum joule-heated

electrons. However, it follows immediately from a turbulent-heating model, in that ions receive their energy directly from fluid kinetic energy, and are insensitive to electron temperature. $T_{c}$ only needs to be high enough to keep the magnetic Reynolds number large, so that B-field energy goes mostly into fluid motion rather than resistive dissipation.

\section{DISCUSSION}

The occurrence of this kind of turbulent heating ought to be a universal property of simple (unstabilized) pinches. However, for scveral reasons one might not have expected it to be a dominant process for pinches formed by the traditional breaking down of a tenuous gas:

1. The explosive radial expansion resulting from turbulent heating would drive a "classical" gas pinch into its chamber walls almost immediately, since the wall/pinch radius ratio for these devices is usually not greater than about ten. It has been observed that such discharges usually disrupt shortly after instabilities appear. The HDZP-II is unique in being so far removed from the chamter wall ( $>10^{4}$ pinch radii) that no plasma-wall encounter can occur; turbulent heating can proceed throughout the current rise.

2. At the very low densities and much larger geometrical scale of gas pinches, the time required for turbulent plasma motion to decay into ion thermal energy is probably not much less than the lifetime of the pinch. As a consequence the process suggested above may not have time in which to achieve a steady state.

3. The effective turbulent resistance, dl/dt, varies as $I / r$. In the HI)7.P.II, this is probably thre .rders larger than in any previous pinch, so that here, uniquely in pinch research, turbulent heating is the dominant process.

'Ihe amalysis presented above is very approximate in most of its details. However, one may be confident about some of its leatures. The radial expulsion of plasma by $\mathbf{m}=\mathbf{0}$ instabilieies, with external circuit reconnection, and isolation and sulseepuent decay of cells of flux and turbulence, is a very likely mechanism. The expression for this effective power input to ions is subject to large uncertaintices in its const:ant multipliers. but the scalling with current and radius is probably corred. The crude simulation employed here would ideally be lollowed by more sophisticated efforts; this maty not be practical, however. becaluse the next step should be a 2 -1) code ilat would smmulate instabilitices in detail, and the cost and time needed to follow the system lhrought hundededs of prowhlo times and over hundreds of original pinch radii could he prohibitive.

To the extent that this model is correct, one mity predict that rappid

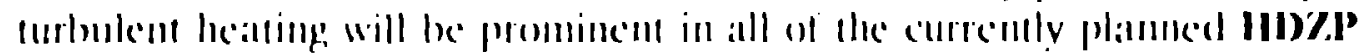


experiments. In cases where the original fiber is significantly larger than the present $30 \mu \mathrm{m}$, thus raising both $\mathbf{r}$ and $\mathbf{N}$, and peak current smaller, tile effect may be somewhat mitigated.

\section{APPENDIX A: DIFFERENTIAL EQUATIONS}

The set of coupled ODE's for the $D_{2}$ fiber sinulation are for seven variables: ion density $\mathbf{n}_{\mathbf{i}}$ (equal to the electron density $\boldsymbol{n}_{\mathbf{e}}$ ), their corresponding energy densities $U_{p} U_{e}$ the pinch radius $r$, radial velocity $v$, integrated neutron yield $\mathbf{N}_{\boldsymbol{m}}$ and the total pinch current $\mathbf{I}$. The deuterium of the pinch is assumed to be fully ionized at the beginning of the calculation, and to have a temperature of $10 \mathrm{eV}$; the starting current is then set at a value that produces dynamic equilibrium at the initial pinch radius.

SI units are used, with the exception that the temperatures are in keV:

$$
\begin{aligned}
& \frac{d n_{i}}{d t}=-\frac{2 v}{r} n_{1} \quad \text { (expansion) }
\end{aligned}
$$

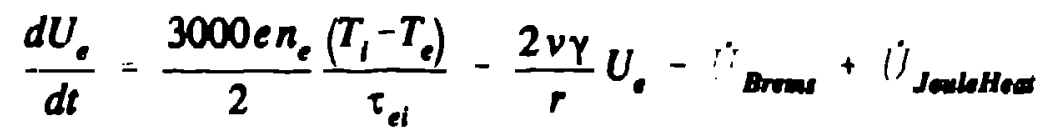

$$
\begin{aligned}
& \dot{U}_{\text {Brow }}=5.3 \times 10^{-37} n_{e}^{2} \sqrt{T_{e}} \quad \dot{U}_{\text {JoulbHow }}=3.3 \times 10^{-10} \frac{I^{2} \ln \Lambda}{r^{4} T_{e}^{3 / 2}} \\
& \frac{d U_{l}}{d t}-\frac{3000 e n_{l}}{2} \frac{\left(T_{e}-T_{i}\right)}{\tau_{e l}}-\frac{2 v \gamma}{r} U_{i}+\dot{U}_{\text {Thritoer }} \\
& \dot{l}_{\text {Turthour }}-1.0 \times 10^{12} \frac{l^{3}}{r^{3} \sqrt{m}} \\
& \frac{d r}{d t}-v \\
& \frac{d v}{d t}-\frac{2 \pi r}{m}\left(P_{i n t} \quad P_{e x}\right)=\frac{2 \pi r}{m}\left(\frac{2}{3} \sum U-\frac{\mu_{0} l^{2}}{8 \pi^{2} r^{2}}\right) \\
& \frac{d N_{n}}{d t} \quad \pi r^{2} C_{p} n_{i}^{2} 0 v
\end{aligned}
$$

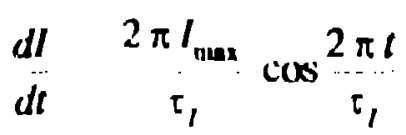


where $\ell_{\mathrm{p}}$ is the pinch length, $5 \mathrm{~cm}$ in the present experiment, and $\mathbf{m}$ in Eqs. (11) and (13) is the total mass/meter.

The D-D reaction rate and particle energy exchange time constant in the above equations are:

$$
\begin{aligned}
& \overline{\sigma v}_{D D}=\frac{2.3 \times 10^{-20}}{T_{i}^{2 / 3}} \exp \left(-\frac{18.76}{T_{i}^{1 / 3}}\right) \frac{m^{3}}{s}, \\
& \tau_{e t}=2.5 \times 10^{19} \frac{T_{3}^{3 / 2}}{n_{e} \ln \Lambda} .
\end{aligned}
$$

In addition, the temperatures and energy densities are related as

$$
T_{e d}=4.16 \times 10^{15} \frac{U_{e d}}{n_{e d}} .
$$

It is assumed that $\gamma=5 / 3$.

Additional simplifying assumptions are made in Eqs (9)-(15) are:

- The plasma is assumed to retain a uniform distribution over $\mathbf{r}$; th : expansion terms in the particle and energy density equations reflect this assumption (0-D self-similarity).

- In Eq. (13), the pinch mass is assumed to reside at the outer boundary.

- Since it is assumed in this calculation that the total burn fraction is too small to produce significant pressure from charged reaction products, equations for their number and energy conservation are not included.

Finally, a small viscosity term was included in Eq. (13) to damp oscillations in the solution near $\mathbf{t}=\mathbf{0}$. It appears to have had no significant effert on later progress of the calculation. Some residual oscillation is still apparent. 